\title{
Biomarkers in Hereditary Angioedema
}

\author{
Grzegorz Porebski ${ }^{1}$ (1) $\cdot$ Mateusz Kwitniewski ${ }^{2}$ (]) $\cdot$ Avner Reshef $^{3}$ (1)
}

Accepted: 24 January 2021 / Published online: 9 February 2021

(c) The Author(s) 2021

\begin{abstract}
A biomarker is a defined characteristic measured as an indicator of normal, biologic, pathogenic processes, or biological responses to an exposure or intervention. Diagnostic biomarkers are used to detect a disease or a subtype of a disease; monitoring biomarkers are measured serially to assess a medical condition; response biomarkers are used to check biologic response following a medical intervention; predictive biomarkers are used to identify patients who are more likely to respond to a medical intervention; and prognostic biomarkers are used to assess the future likelihood of a clinical event. Although biomarkers have been extensively investigated and validated in many diseases and pathologies, very few are currently useful for the diagnosis, evaluation of disease activity, and treatment of hereditary angioedema (HAE). Pathophysiologic pathways involved in HAE reveal a plethora of molecules from the complement, coagulation, and fibrinolysis systems or from the vascular endothelium, which may serve as biomarkers. The most promising candidates, together with their laboratory readout systems, should be evaluated with regard to their analytical and clinical validity and utility. To be highly specific, such biomarkers should be linked to the pathomechanisms of HAE, particularly the bradykinin-generating cascade. Additionally, major advances in high-throughput omics-based technologies may facilitate the discovery of new candidate biomarkers in the future. This review will cover the existing as well as future potential biomarkers that will support the diagnosis, monitor disease activity, and can be used to assess the efficacy of new avenues of therapy of HAE and other forms of angioedema.
\end{abstract}

Keywords Hereditary angioedema $\cdot$ Biomarkers $\cdot \mathrm{C} 1$ inhibitor $\cdot$ Diagnosis $\cdot$ Management

\section{Introduction}

Biomarkers are currently one of the most extensively investigated areas of biomedical sciences, as reflected by search results in major public databases. A search for the term "biomarker" yields over 1.5 thousand active and recruiting studies in a web-based registry of clinical trials [1]. Moreover, this keyword generates over 40,000 results in the PubMed search (filters applied: clinical trial, humans) [2]. In the field of allergy and clinical immunology, biomarkers have been recently studied in numerous diseases, such as atopic dermatitis [3], allergic

Grzegorz Porebski

g.porebski@uj.edu.pl

1 Department of Clinical and Environmental Allergology, Jagiellonian University Medical College, Krakow, Poland

2 Department of Immunology, Faculty of Biochemistry, Biophysics and Biotechnology, Jagiellonian University, Krakow, Poland

3 Barzilai University Medical Centre, Ashkelon, Israel rhinitis [4], bronchial asthma [5, 6], food allergy [7], and severe drug hypersensitivity reactions [8]. Most commonly, investigators have searched for new biomarkers that would predict disease severity $[3,5]$, determine the risk of severe reactions $[7,8]$, or discriminate between disease endotypes [6].

The main focus of the present review is hereditary angioedema (HAE). The most frequent type of HAE is characterized by recurrent episodes of tissue swelling due to low production or nonfunctional serine protease inhibitor, namely, $\mathrm{C} 1$ esterase inhibitor (C1-INH). Affected enzymatic pathways include the classic complement cascade, fibrinolytic pathway, and contact activation system responsible for bradykinin formation. The loss of the inhibitory activity of C1-INH leads to bradykinin overproduction, resulting in vascular instability in the endothelial wall, followed by hyperpermeability and plasma extravasation [9]. Another types of HAE have a similar clinical picture, but with normal C1-INH level and activity and a different genetic background, namely, mutations in the gene-encoding coagulation factor XII (FXIIHAE), plasminogen (PLG-HAE), angiopoietin-1 (ANGPT1HAE), or an unknown mechanism (U-HAE) [10]. 
The aim of this article is to review biomarkers that could be used for diagnosis, evaluation of disease activity, and management of these clinical entities.

Scientific literature offers numerous definitions and classifications of biomarkers. To ensure effective communication and consistent use of key terms, we followed the nomenclature proposed by the FDA-NIH Biomarker Working Group [11]. Hence, biomarker was considered as "a defined characteristic that is measured as an indicator of normal biological processes, pathogenic processes, or biological responses to an exposure or intervention, including therapeutic interventions" [11]. For the purpose of this review, we also used the following terms: diagnostic biomarker (used to detect a disease or a subtype of a disease), monitoring biomarker (measured serially to assess the medical condition of interest), response biomarker (used to check if a biological response has occurred in a patient who underwent a medical intervention), predictive biomarker (used to identify patients who are more likely to respond to a medical intervention), and prognostic biomarker (used to assess the likelihood of a clinical event in the future). Some of these biomarker categories may overlap in particular clinical situations [11]. An ideal biomarker should demonstrate evidence for strong analytical validity and clinical utility, reflect a pathophysiology of a disease, with proven feasibility (an easy and reliable measurement method), and either no or low invasiveness, which implies an easy access to biological material (e.g., blood, saliva, or urine) $[12,13]$. The accepted approach to biomarker definition entails the selection of some candidate indicators that are associated with the clinical or pathophysiological aspects of a disease. However, with the advent of novel high-throughput techniques, it is now possible to perform non-targeted multi-omics analyses (e.g., genomic, transcriptomic, proteomic, or metabolomic) to identify a cohort of biomarkers for further in-depth research [12].

In daily practice, clinicians taking care of patients with HAE are faced with several challenges, such as (i) large inter- and intra-familial heterogeneity in the clinical manifestations of the disease; (ii) early diagnosis of unpredictable oncoming angioedema attacks; (iii) a differential diagnosis of HAE subtypes; and (iv) objective assessment of disease severity, which may guide therapeutic decisions. These challenges are the driving force behind the current efforts to identify measurable biomarkers of HAE. Finding such biomarkers could facilitate the diagnosis and prediction of an upcoming attack, as well as help to monitor disease activity, discriminate between high and low responders to a specific drug, or select the best candidates for a given treatment.

This review represents a clinician's perspective and constitutes an attempt to answer the question: to what extent biomarker discovery can help overcome the above challenges? In the broadest sense, any measurable parameter may serve as a biomarker, including clinical features or radiographic findings, but the scope of this review is to discuss only potential laboratory biomarkers that involve essential biological processes, from nucleic DNA to mediators of effector mechanisms.

\section{Biochemical Biomarkers}

HAE involves numerous processes in pathophysiologic pathways, during remissions and attacks. Recently, plasma enzymatic cascade systems, endothelium-derived factors, and inflammatory mediators have been extensively studied with regards to the mechanistic contributions to the pathology of HAE. Some have important implications for the discovery of biomarkers that could be used in the diagnosis and monitoring [13-17]. However, only a few laboratory tests (antigenic and functional C1-INH, complement C4) with established threshold values are currently used in clinical practice for diagnosis and decision-making [14] (Table 1).

Candidate biomarkers, discussed in detail below, were selected based on the current understanding of the biochemical processes underlying HAE as well as on the evidence for their relevance in clinical disease manifestations.

\section{Complement Cascade Biomarkers}

Functional C1-INH (fC1-INH, C1-INH activity) plasma levels are considered a reference test in the diagnosis of HAE due to C1-INH deficiency (C1-INH-HAE), particularly of type $2[14,18-20]$. However, there have been some concerns in relation to this test, including variability due to sample handling and time of storage or an increase in its levels in the course of inflammation and infection [21-23]. In addition, different types of assays may yield divergent results [19, 24]. Moreover, fC1-INH measurements can be affected by the presence of autoantibodies to C1-INH in the sample [16], as well as by danazol treatment [25] or plasma-derived C1-INH [26]. Currently, fC1-INH levels are measured by a commercial chromogenic assay or an enzyme-linked immunosorbent assay (ELISA), which detects complexes formed by $\mathrm{C} 1-\mathrm{INH}$ and complement $\mathrm{C} 1 \mathrm{~s}$, activated FXII, or plasma kallikrein (PKa) [18, 21, 27]. However, functional assays have limited ability in detecting small changes over time $[18,21]$. Recently, a highly robust point-ofcare test to measure fC1-INH in dried blood spot has been described [28]. It is based on the quantitation of the enzyme reaction product by liquid chromatography-tandem mass spectrometry and is characterized by high reproducibility 
and accuracy, together with sample storage stability. Whereas the diagnostic value of functional C1-INH levels is well established, its value as a monitoring biomarker remains unclear. A predominant opinion is that the level of fC1-INH has little relationship to the clinical course of C1-INH-HAE $[13,14]$. Suffritti et al. showed lower fC1-INH in patients during attacks than during remission ( 31 and 131 patients, respectively) [29]. However, Kajdasci et al. and Cugno et al. did not confirm this finding in their studies involving 18 and 28 patients with C1-INH-HAE, respectively [30, 31]. Other studies focused on associations between C1-INH function and disease course. Kelemen et al. demonstrated that the baseline level of fC1-INH correlates with the HAE severity score [32]. In a subsequent report by the same group, lower fC1-INH levels were observed in patients with a higher number of attacks and a higher need for C1-INH on-demand treatment [33]. In turn, Bafunno et al. could not find a significant correlation between fC1-INH level and disease severity score or age of onset [34]. Further insight was provided by clinical trials with plasma-derived C1-INH supplementation. A level of approximately $40 \%$ of fC1-INH appears to protect against angioedema attacks in most patients who received prophylactic treatment with subcutaneous C1-INH [35], which is in line with previous clinical observations [36, 37]. Therefore, these ranges of fC1-INH may serve as a prognostic biomarker of disease activity, i.e., assessment of likelihood of future attacks.

Antigenic Cl-INH ( $\mathrm{AgCl}$-INH) plasma concentration is a critical diagnostic biomarker for the diagnosis of C1-INHHAE type 1 [38]. Its concentration can be measured by nephelometry, turbidimetry, or radial immunodiffusion, depending on local availability and cost [23]. The results may be influenced by replacement therapy with C1-INH [14]. In principle, $\mathrm{AgC1}$-INH is not considered a valuable monitoring biomarker of the clinical course of the disease [13, 14, 30, 31]. However, Spath et al. demonstrated most frequent attacks in patients with C1-INH-HAE when AgC1INH levels were below $0.035 \mathrm{~g} / 1$ [39]. Other authors reported that $\mathrm{AgC1}$-INH levels were lower during attacks [29] or negatively correlated with the annual number of attacks [33].

The protease-inhibitor complex Cl-INH-Cl(r,s) reflects contact system activation and thus may be considered a potential biomarker [14]. Its plasma concentration, which can be determined by ELISA, is influenced by the amount of C1-INH present in plasma and is artificially low in C1-INH deficiency [14, 33]. Plasma levels of C1-INH$\mathrm{C} 1(\mathrm{r}, \mathrm{s})$ complexes were found to be higher in patients with C1-INH-HAE than in healthy controls $[33,40]$ and increased further during angioedema attacks [41]. Patients with higher $\mathrm{C} 1-\mathrm{INH}-\mathrm{C} 1(\mathrm{r}, \mathrm{s})$ levels had a history of more severe attacks and more often required emergency treatment [40]. Plasma C1-INH-C1(r,s) complex levels were reported to normalize in patients treated with stanozolol along with a reduction in symptoms [42], thus showing promise as a biomarker for monitoring therapeutic response (a response biomarker) [43].

Complement $\mathrm{C} 4$ serves as an important contributory diagnostic biomarker in C1-INH-HAE [14, 38], because its level is reduced in most patients, especially during attacks $[16,24]$. Nevertheless, its performance as a monitoring biomarker is poor. Complement $\mathrm{C} 4$ was shown to correlate with the frequency of attacks and on-demand consumption of C1-INH concentrate [33] but not with disease severity scores [32]. No significant difference was observed in $\mathrm{C} 4$ levels between remission and acute abdominal attacks [31].

Additional laboratory complement indices were also reported in HAE. Varga et al. found that anti-Cl-INH IgM antibody levels correlated with disease severity in C1-INH concentrate-naive patients [44]. Other reports indicated that the levels of Mannose-binding lectin-associated serine proteases (MASP-1, MASP-1)-C1-INH complexes are lower in C1-INH-HAE patients and correlate with the frequency of attacks [45], whereas the levels of MASP-2 and ficolin-3/ MASP-2 complexes increase during attacks [46] (Table 1).

\section{Contact System and Bradykinin-Forming Cascade Biomarkers}

Since bradykinin $(B K)$ is the major mediator of swellings in HAE $[13,47]$, it is expected to be the most accurate biomarker of upcoming attacks. Indeed, plasma BK levels were reported to be higher in patients with C1-INHHAE than in healthy controls, with a further significant rise during attacks [48]. Moreover, they were found higher in blood taken from the site of angioedema than at a control site in classical report by Nussberger et al. [49]. Nevertheless, clinical utility of plasma BK levels is being questioned because of its high sensitivity to preanalytical procedures and a very short half-life (measured in seconds) $[13,14]$. In addition, the measurement of BK and its breakdown metabolites, such as des-Arg-BK, based on liquid chromatography with mass spectrometry, is technically challenging $[50,51]$. The clinical utility of a commercially available assay kit for detecting the product of BK degradation, which might be a surrogate for BK quantification, awaits confirmation [13].

High molecular weight kininogen $(H K)$ proteolysis by active PKa results in generation of cleaved $H K(c H K)$ and $\mathrm{BK}$. Thus, cHK is thought to be a promising indirect marker of BK release and contact system activation, which occurs during HAE attacks. Western blotting for cHK detection is hard to standardize and subjective in interpretation. Semiquantification of results can be done by scanning gels [13]. New effective methods for cHK measurement are based on a monoclonal antibody ELISA [52] and modification of liquid chromatography-mass spectrometry [53]. Cugno 
et al. observed, in patients with C1-INH-HAE, high levels of cHK during attacks and normal levels during remission [54, 55]. In a subsequent report on a large population of C1-INHHAE patients, Suffritti et al. reported cHK levels to be higher in patients than in controls and to further increase during attacks. They also demonstrated that $\mathrm{cHK}$ levels were higher in highly symptomatic patients in comparison with those with less frequent attacks [29]. Using an immunoassay based on ELISA, Hofman et al. confirmed that cHK levels were elevated in patients with C1-INH-HAE during remission and further increased during attack [52]. Banerji et al. showed significant reductions in cHK levels in C1-INH-HAE patients treated with $300 \mathrm{mg}$ and $400 \mathrm{mg}$ of lanadelumab, a monoclonal antibody against PKa [56]. In turn, Bova et al. measured cHK levels in a cohort of $105 \mathrm{HAE}$ patients with normal C1-INH levels (nC1-INH-HAE), including U-HAE and FXII-HAE [57]. cHK was measured during remission in plasma collected with and without using protease inhibitors. In patients with U-HAE, cHK levels were similar to those in healthy controls with the use of protease inhibitors and significantly increased without them. In patients with FXIIHAE without the use of protease inhibitors, cHK levels were higher than in controls and similar to those observed in patients with U-HAE [57].

Therefore, cHK levels can discriminate between patients and healthy individuals, between highly symptomatic and less symptomatic patients, and between acute attack and remission. The development of a new reliable and less laborious measurement method may facilitate the clinical application of this parameter.

Plasma kallikrein $(\mathrm{PKa})$ is also considered a potential biomarker of BK-mediated angioedema attacks. Spontaneous PKa activity, measured with the use of a chromogenic substrate, was higher in patients with C1-INHHAE than in controls and further increased during attacks [29]. Similar results were reported by other authors [58]. Lara-Marquez et al. developed an assay based on plasma capacity to generate active PKa under ex vivo stimulation with dextran sulfate [59]. Using different threshold cutoff values in this assay, patients with BK-dependent angioedema (nC1-INH-HAE and C1-INH-HAE) could be distinguished from controls without swelling attacks and patients with histaminergic angioedema, based on a higher amount of generated PKa [59].

Activated coagulation factor FXII ( FXIIa) was shown to be higher in patients with C1-INH-HAE than in healthy controls, with a further increase during attacks [54, 58, 60]. The activity of FXII was also evaluated as a possible biomarker of FXII-HAE in symptom-free periods, but studies provided inconsistent results, with some suggesting an increase while others reporting no difference between patients and healthy controls $[61,62]$.
Factor XIIa/C1-INH complexes were studied by Konings et al. [63]. They showed that the levels of these complexes, as well as the levels of FXIa-C1INH and PKa-C1INH complexes, were lower in patients with C1-INH-HAE than in healthy controls after an in vitro activation of the samples with an FXII trigger.

Kinins - degradation and accumulation of kinins, including BK, may modify the clinical phenotype of HAE. Carboxypeptidase $N(C P N)$, angiotensin-converting enzyme $(A C E)$, and aminopeptidase $P(A P P)$ are major kinases involved in kinin catabolism [64]. Drouet et al. investigated the kininase activity in C1-INH-HAE patients with and without androgen prophylaxis [65]. APP levels were inversely correlated with disease severity in all patients, and CPN activity showed the same relationship only in untreated patients. Subsequently, a similar study was conducted in patients with FXII-HAE, showing an inverse correlation between the disease severity score and both ACE and CPN activities, but not APP [66]. Moreover, the total activity of serine proteases was shown to be higher in patients with C1-INH-HAE and nC1-INH-HAE than in healthy controls and to further increase in those with nC1-INH-HAE during attacks [67].

\section{Coagulation and Fibrinolytic Pathway Biomarkers}

Coagulation and fibrinolysis are also pathways activated in HAE and therefore extensively investigated for potential biomarkers. To date, a wide range of fibrinogen split products in patients during HAE attack and remission have been studied [31, 55, 60, 68, 69]. The findings from these studies are summarized in Table 1.

Plasminogen activator inhibitor (PAI)-1 levels, prothrombin time, and activated partial thromboplastin time were also found to be lower during C1-INH-HAE attacks compared with a symptom-free period [60]. The PAI-1 level was also found lower in patients with U-HAE and FXII-HAE compared with control individuals, but the difference was not significant [70]. In the same study, also PAI-2 levels were reported to be lower in patients with U-HAE and FXII-HAE than in controls, but this finding was not corroborated by other studies [71].

$D$-dimer levels were also found elevated and may also discriminate between abdominal HAE attacks and abdominal colic episodes [31, 72], between multiple- and singlesite attacks [60], and between submucosal (abdominal, oropharyngeal-laryngeal) and subcutaneous (peripheral, facial) attacks [73]. D-dimer levels were observed to decrease at day 7 after attack $[72,73]$. Prophylaxis with continuous supplementation of subcutaneous plasma-derived C1-INH seems to suppress D-dimer levels, in parallel with a significant reduction in the rate of HAE attacks [74]. 
Collectively, laboratory parameters associated with coagulation and fibrinolysis may have limited usefulness as biomarkers because of their interpatient variation in the plasma [13]. They can be useful to some extent for disease course monitoring when compared with baseline values of the same individual. Another major disadvantage that limits their potential use as biomarkers is the fact that they may not be specific for HAE. For instance, a D-dimer test is widely used and easily available in emergency settings, but D-dimer levels are elevated in numerous medical conditions, i.e., pulmonary embolism and venous thromboembolism, but also in infection, autoimmune disorders, malignancy, as well as in smokers, pregnant women, and elderly individuals [75].

\section{Endothelium-Associated Biomarkers}

As the endothelium is inherently related to microvascular permeability and, in consequence, the swelling phenomenon, it represents another area of interest regarding potential biomarkers in HAE. Studies in this field involved vascular endothelial cadherin (transmembrane adhesive protein) [72, 76], von Willebrand factor (marker of endothelial damage), soluble E-selectin (cytokine-induced adhesion molecule), endothelin-1 (vasomotor activity regulator) [30, 77, 78], arginine vasopressin, adrenomedullin [78], atrial natriuretic peptide [79], as well as endothelialderived endocan and vascular cell adhesion molecule-1 (markers of endothelial function) [80]. The subsequently studied modulators of vascular permeability included vascular endothelial growth factors, angiopoietin-1 (which promotes endothelial stabilization) and angiopoietin-2 (which facilitates vascular permeability) [81, 82], secreted phospholipases A2 (particularly the 2A group) [83], and platelet-activating factor acetylhydrolase [81]. Studies investigated changes in the above factors in small groups of patients with C1-INH-HAE during attacks and symptomfree periods, in comparison with healthy controls, as shown in Table 1. More recent research also involved patients with nC1-INH-HAE in remission. Bova et al. reported increased levels of angiopoietin-1 and vascular endothelial growth factors $A$ and $C$ in patients with U-HAE as well as increased levels of vascular endothelial growth factor $C$ in patients with FXII-HAE [57].

It is well known that stimulation of endothelial cells with BK and its analogs causes the release of prostacyclin and unstable endothelium-derived relaxing factor [84], which was subsequently identified as nitric oxide species contributing to enhanced vascular permeability [85]. Bas et al. investigated the long-lived metabolite of prostacyclin, namely, 6-keto-prostaglandin F1- $\alpha$, as a potential biomarker for the diagnosis of angioedema induced by angiotensin-converting enzyme inhibitors [86]. In turn, Demirturk et al. showed that plasma levels of endothelial nitric oxide synthase were significantly higher in patients with C1-INH-HAE in remission and during attacks than in healthy participants and that the levels of nitric oxide metabolites were elevated only during attacks [87]. Further research involved also the effect of HAE on endothelial function expressed by blood concentrations of asymmetric dimethylarginine, a strong inhibitor of nitric oxide synthesis associated with numerous common conditions, such as atherosclerosis. In a group of HAE patients (24 with C1-INH-HAE and 14 with FXII-HAE), asymmetric dimethylarginine levels were higher than in controls [88]. In a subsequent report, this group demonstrated that serum concentrations of advanced oxidation protein products, used as markers of oxidative stress, were higher in patients with C1-INH-HAE and FXII-HAE during remission than in controls [89]. In another study investigating oxidative stress in HAE, the authors showed that the levels of reactive oxygen species in peripheral blood mononuclear cells of patients with C1-INH-HAE were higher than in controls; however, no differences were observed between the groups in plasma levels of advanced oxidation protein products [90].

\section{Other Areas of Research}

A number of studies have investigated the association of HAE with low-grade inflammation markers, immune system elements, and hormones. Investigators compared patients with C1-INH-HAE and healthy controls with respect to a number of factors, including $C$-reactive protein, erythrocyte sedimentation rate, white blood cell, and neutrophil counts [91-93], a wide network of pro-inflammatory and antiinflammatory cytokines (i.e., interleukins $I L-1 \beta, I L-2, I L-4$, $I L-5, I L-6, I L-8, I L-10, I L-13, I L-17$, interferon- $\gamma$, tumor necrosis factor- $\alpha$, granulocyte colony-stimulating factor, and granulocyte-macrophage colony-stimulating factor) [87, 94, 95], as well as sex hormones, such as progesterone, together with sex hormone-binding globulin [96]. Differences in these factors between patients during remission and attacks, as well as in comparison with healthy controls, are summarized in Table 1. More recently, significant changes were shown in multifunctional human glycoprotein fetuin-A levels between symptom-free periods and attacks in patients with C1-INHHAE [93]. Moreover, fragmentation patterns of serum glycoprotein 120 were also reported as a potentially useful biomarker in patients with nC1-INH-HAE [97] (Table 1).

The utility of biomarkers reported in the above studies should be interpreted with caution if (i) biochemical parameters investigated in the study are involved also in other clinical pathologies (low specificity in HAE, uncertain direction of a causative relationship between the studied laboratory parameter, and HAE); (ii) the study includes a small sample size of patients; and (iii) results of laboratory measurements are highly variable. For biomarkers expected 
to facilitate the monitoring of HAE severity or response to treatment, well-defined endpoints are necessary. However, differences in symptom severity scores used and lack of standardization make it difficult to compare the results between studies. In turn, potential diagnostic biomarkers for HAE or angioedema attacks require a gold standard for their validation. Hence, a new test based on the measurement of a given molecule has to be validated against proven clinical events or cases (e.g., an investigator-confirmed attack or a known genetic mutation with proven pathogenicity). Studies designed to elucidate the mechanisms of HAE usually are unable to strictly follow the above requirements for biomarker identification, but there are already some ongoing trials directly dedicated to the study of biomarkers in HAE, including specific biomarkers of BK-mediated angioedema attack in a pediatric population, potential disease-specific biomarkers for HAE with the use of dry blood spot technology, and, finally, the use of cHK as a biomarker in C1-INH-HAE [1].

\section{Genomic Biomarkers}

Genomic biomarkers include DNA sequence variations, such as single-nucleotide variants, insertions, and deletions, as well as RNA alterations, such as differential gene expression and micro RNAs [98]. Genetic testing of angioedema is primarily focused on detecting alterations in the DNA of genes encoding proteins that are part of the complement, fibrinolysis, coagulation, kinin, and vasculature systems, including C1-INH (SERPING1), FXII (F12), plasminogen $(P L G)$, or angiopoietin-1 (ANGPT1). Genomic biomarkers are mainly employed to support the diagnostic workup for HAE, but efforts are being made to identify correlations of the detected genetic changes with disease severity and treatment outcomes (prognostic or predictive biomarkers) [99].

\section{Diagnostic Applications of Genetic Biomarkers}

The most common form of hereditary angioedema, C1-INH-HAE, is primarily caused by alterations in the SERPINGl gene. Nevertheless, SERPING1 genotyping is not recommended for the diagnosis of C1-INH-HAE, with some exceptions (e.g., newborns or inconclusive biochemical tests), because biochemical C1-INH testing is cost-effective and reliable [100, 101].

Genotyping is required for the diagnosis of nC1-INHHAE [100, 102]. It is a rare type of HAE associated with mutations in F12 [103], PLG [104], and ANGPT1 [105] genes. However, it is estimated that the genetic background of approximately $70 \%$ of patients with nC1-INH-HAE is unknown [106]. The F12 gene, encoding human FXII, is located on chromosome 5 and consists of 13 introns and 14 exons [107]. To date, four $F 12$ alterations have been linked to nC1-INH-HAE, and all are located on exon 9. Two missense mutations result in the substitution of threonine to arginine or lysine at position 309 of FXII (p.Thr309Lys/Arg) [103]. These two mutations are responsible for approximately $15 \%$ to $30 \%$ of nC1-INH-HAE cases [100]. In addition, a deletion of $72 \mathrm{bp}$ (c.971_1018+24del72) [108] and a duplication of 18 bp (c.892_909dup) [109], affecting the same region of the FXII protein, were identified. Therefore, only exon 9 of F12 should be investigated as a routine molecular diagnostic biomarker of FXII-HAE [100].

More recently, new mutations in ANGPTI [105, 110], $P L G$ [104, 111], KNG1 (Kininogen 1) [112], and MYOF (myoferlin) [113] genes were reported in nC1-INH-HAE. However, the importance of these genetic changes in the pathophysiology of HAE is not yet well understood. Several loss-of-function mutations have been identified in the ANPGPT1 gene-encoding angiopoietin-1 (ANGPT1HAE). The p.Ala119Ser substitution affects binding of the protein to the tyrosine kinase Tie 2 receptor on endothelial cells [105], and, in turn, may reduce the ability to counteract the changes in vascular permeability induced by a variety of mediators, including BK or vascular endothelial growth factor [110]. Other potentially pathogenic variants of ANGPT1 (p.Ala8Val; p.Gln370His) were found by Cagini et al. [114]; however, more evidence supporting these preliminary results is needed. To date, one missense mutation in the $P L G$ gene, which encodes plasminogen, has been linked with nC1-INH-HAE (PLG-HAE). The substitution of lysine to glutamic acid at position 311 of mature plasminogen protein was described independently by Bork et al. [104] and Dewald et al. [111]. It was suggested that altered structure of plasminogen may affect the affinity to its binding partners [111]. The links between plasminogen activation and angioedema were revised in detail by Maas [115]. The coincidence of multiple mutations in the genes of the complement, fibrinolysis, coagulation, and kinin systems, as well as their role in HAE development, is still the subject of debate. Patients harboring both SERPINGI and PLG mutations were described by Bork et al. [116]. Therefore, there is a strong need for a polygenic diagnosis of nC1-INH-HAE.

For some time now, whole-exome sequencing (WES) has been widely used to uncover a molecular background and complex genetic interactions in nC1-INH-HAE. Bork et al. [112] applied it to identify a novel variant of the $K N G 1$ gene, resulting in the substitution of methionine to lysine at position 379 of the high-molecular-weight kininogen protein (p.Met379Lys). The amino acid change may affect the formation of $\mathrm{BK}$ from this protein and was considered to be 
Table 1 Laboratory measures discussed in the review, which are considered as potential biochemical HAE biomarkers in literature

\begin{tabular}{|c|c|c|c|c|c|}
\hline System involved ${ }^{\mathrm{a}}$ & $\begin{array}{l}\text { Correlation with disease } \\
\text { severity }^{\mathrm{b}}\end{array}$ & $\begin{array}{l}\text { Increase or further } \\
\text { increase during attacks } \\
\text { (vs during remission) }\end{array}$ & $\begin{array}{l}\text { Higher level in remission } \\
\text { (vs healthy controls) }\end{array}$ & $\begin{array}{l}\text { Lower level in remission } \\
\text { (vs healthy controls) or } \\
\text { decrease during attacks } \\
\text { ( } \downarrow)\end{array}$ & References \\
\hline Complement & $\begin{array}{l}\text { C1-INH function } \\
\text { C1/C1-INH complex } \\
\text { Anti-C1-INH IgM } \\
\quad \text { antibodies } \\
\text { MASP-1 } \\
\text { MASP-1/C1-INH } \\
\text { complex }\end{array}$ & $\begin{array}{l}\text { C1/C1-INH complex } \\
\text { MASP-2 } \\
\text { MASP-2/ficolin-3 } \\
\quad \text { complex }\end{array}$ & C1/C1-INH complex & $\begin{array}{l}\text { C1-INH function } \\
\text { C1-INH concentration } \\
\text { C4 } \\
\text { MASP-1 } \\
\text { MASP-1/C1-INH } \\
\quad \text { complex }\end{array}$ & $\begin{array}{l}{[14,16,18-2133-38,40-} \\
\quad 42,44-46]\end{array}$ \\
\hline $\begin{array}{l}\text { Contact activation and } \\
\text { bradykinin }\end{array}$ & $\begin{array}{l}\text { Cleaved HK } \\
\text { APP } \\
\text { ACE and CPN (3) }\end{array}$ & $\begin{array}{l}\text { Bradykinin } \\
\text { Cleaved HK } \\
\text { Plasma kallikrein } \\
\text { Factor XIIa }\end{array}$ & $\begin{array}{l}\text { Bradykinin } \\
\text { Cleaved HK (1)(2) } \\
\text { Plasma kallikrein } \\
\text { Factor XIIa }\end{array}$ & & $\begin{array}{l}{[30,48,52,54,55,57,58,} \\
\quad 60,65]\end{array}$ \\
\hline $\begin{array}{l}\text { Coagulation and } \\
\text { Fibrinolysis }\end{array}$ & & $\begin{array}{l}\text { D-dimer } \\
\text { Prothrombin fragments } \\
\quad 1+2 \\
\text { TAT, PAP complexes } \\
\text { Thrombin, TAFI } \\
\text { PAI-1 }\end{array}$ & $\begin{array}{l}\text { D-dimer } \\
\text { Prothrombin fragments } \\
\quad 1+2 \\
\text { TAT, PAP complexes } \\
\text { Factor XIa }\end{array}$ & $\begin{array}{l}\text { PAI-1 } \downarrow \\
\text { Prothrombin time } \downarrow \\
\text { aPTT } \downarrow\end{array}$ & {$[32,55,60,68,69,72-74]$} \\
\hline Endothelium factors & $\begin{array}{l}\text { VEGF-A }{ }^{\mathrm{c}}, \text { VEGF-C } \\
\text { ANGPT2 }^{\mathrm{c}}\end{array}$ & $\begin{array}{l}\text { VE-cadherin (soluble } \\
\text { form) } \\
\text { VWF antigen } \\
\text { VWF collagen-binding } \\
\text { activity } \\
\text { Soluble E-selectin } \\
\text { Endothelin-1 } \\
\text { Arginine vasopressin } \\
\text { Adrenomedullin } \\
\text { ANGPT1 } \\
\text { eNOS, NO metabolites } \\
\text { ADMA (4) }\end{array}$ & $\begin{array}{l}\text { Soluble E-selectin } \\
\text { Endocan (soluble form) } \\
\text { VCAM-1 (soluble form) } \\
\text { sPLA }_{2} \text { activity } \\
\text { VEGF-A (1), VEGF-C } \\
\quad \text { (1)(2) } \\
\text { ANGPT1 (1) } \\
\text { ANGPT2 } \\
\text { PAF-AH } \\
\text { eNOS } \\
\text { AOPPs (2) } \\
\text { ROS }\end{array}$ & $\begin{array}{l}\text { Atrial natriuretic peptide } \\
\text { ANGPT2/ANGPT1 } \\
\text { ratio } \downarrow \\
\text { sPLA }_{2} \text { activity } \downarrow \\
\text { PAF-AH } \downarrow\end{array}$ & {$[31,72,77-83,87-90]$} \\
\hline Other & Progesterone, SHBG & $\begin{array}{l}\text { CRP, ESR, WBC } \\
\text { Neutrophil count, } \\
\text { neutrophil elastase, } \\
\text { myeloperoxidase, } \\
\text { pentraxin } 3 \\
\text { Fetuin-A } \\
\text { TNF- } \alpha \\
\text { IL-1 } \beta, \text { IL-4, IL-5, IL-6, } \\
\text { IL-8, IL-13, IL-17 } \\
\text { FGFb, G-CSF, GM-CSF }\end{array}$ & $\begin{array}{l}\text { WBC } \\
\text { Neutrophil count } \\
\text { GM-CSF, FGFb, IL-17 } \\
\text { sgp120 fragmentation } \\
\text { (5) }\end{array}$ & $\begin{array}{l}\text { Fetuin-A } \\
\text { TNF- } \alpha\end{array}$ & {$[87,91-97]$} \\
\hline
\end{tabular}

Data in the table concern HAE, unless otherwise indicated: (1) also in U-HAE, (2) also in FXII-HAE, (3) in FXII-HAE, (4) in group consisting from C1-INH-HAE and FXII-HAE patients, (5) also in nC1-INH-HAE incubated at $4{ }^{\circ} \mathrm{C}$ in plastic. Italics: currently in use diagnostic biomarkers; $\downarrow$ decrease during attacks

$A C E$ angiotensin-converting enzyme, ADMA asymmetric dimethylarginine, ANGPTs angiopoietins, AOPPs advanced oxidation protein products, $A P P$ aminopeptidase $\mathrm{P}, a P T T$ activated partial thromboplastin time, C1-INH C1-inhibitor, $H K$ high molecular weight kininogen, $C P N$ carboxypeptidase N, CRP C-reactive protein, eNOS endothelial nitric oxide synthetase, E-selectin endothelial selectin, ESR erythrocyte sedimentation rate, $F G F b$ basic fibroblast growth factor, $G$-CSF granulocyte colony stimulating factor, $G M-C S F$ granulocyte-macrophage colony stimulating factor, ILs interleukins, MASP mannose-binding lectin-associated serine protease, $N O$ nitric oxide, PAF-AH plateletactivating factor acetylhydrolase, PAI-1 plasminogen activator inhibitor-1, PAP plasmin-anti-plasmin complexes, ROS reactive oxygen species, sgp120 serum glycoprotein 120, SHBG sex hormone-binding globulin, sPLA2 secreted phospholipase A2, TAFI thrombin-activatable fibrinolysis inhibitor, TAT thrombin/antithrombin complex, TNF tumor necrosis factor, VCAM vascular cell adhesion molecule, VE-cadherin vascular endothelial cadherin, VEGF vascular endothelial growth factor, VWF von Willebrand factor, WBC white blood cell count

${ }^{\text {a } P a t h o p h y s i o l o g i c ~ p a t h w a y s ~ o f ~ t h e ~ s y s t e m s ~ m a y ~ p a r t i a l l y ~ o v e r l a p ~}$

${ }^{b}$ Disease severity is defined in different ways in particular publications

${ }^{c}$ Higher plasma levels in patients with $>12$ attacks/year than in other patients 


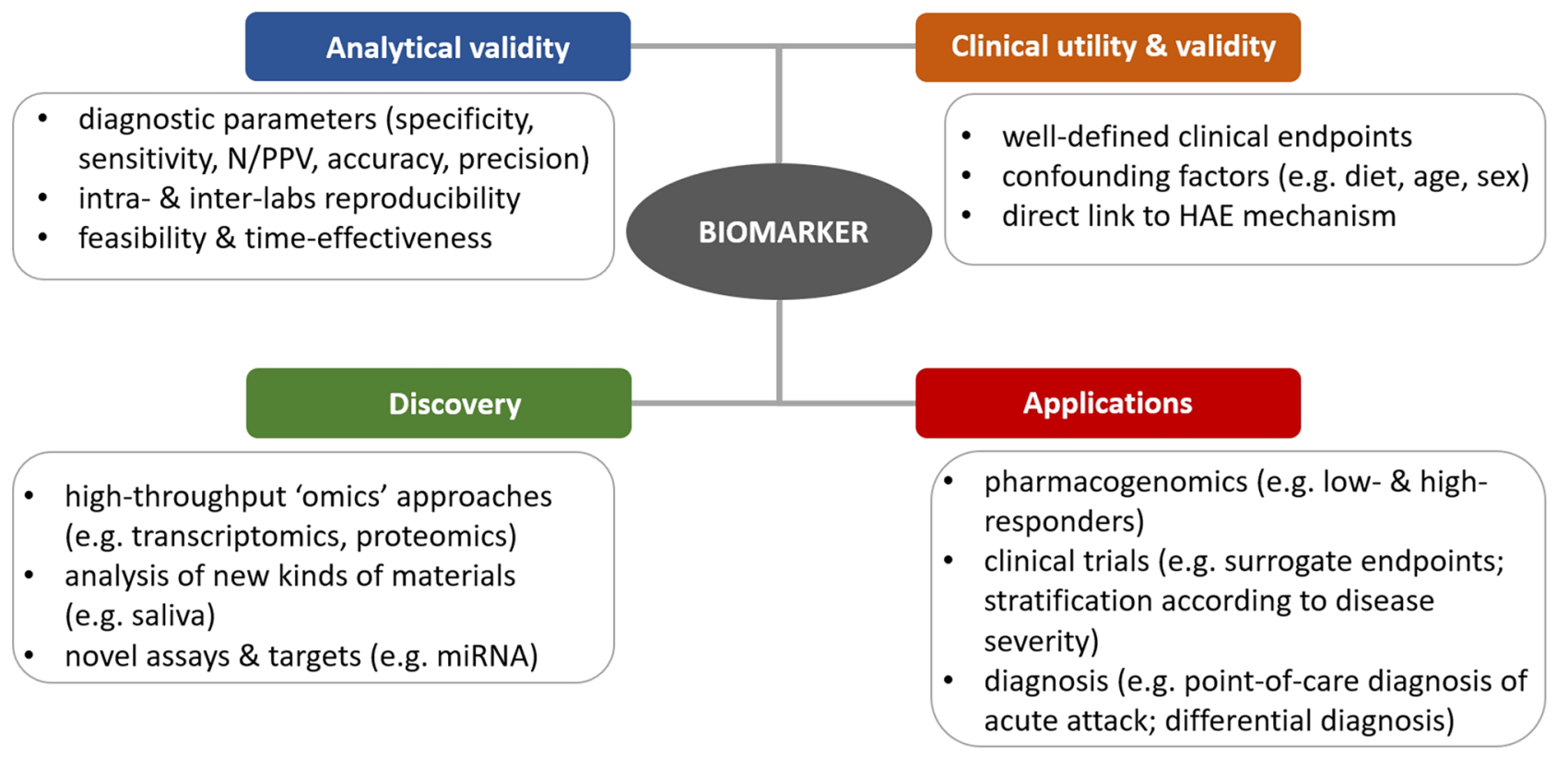

Fig. 1 Biomarkers in hereditary angioedema-future applications and research directions. NPV/PPV, negative/positive predictive value; HAE, hereditary angioedema; miRNA, micro RNA

likely pathogenic. WES has been recently used in multiple studies, and myoferlin (MYOF; p.Arg217Ser) [113] has been linked with nC1-INH-HAE. However, further studies are needed to elucidate the role of these genes in HAE and their potential use as diagnostic biomarkers. To date, no specific guidelines for the diagnosis of the above types of angioedema have been developed. The exclusion of the above pathogenic mutations in patients with normal fC1-INH levels makes it possible to establish the diagnosis of U-HAE and nonhistaminergic angioedema [100].

\section{Prognostic Applications of Genetic Biomarkers}

The severity and course of HAE may vary greatly even among family members harboring the same mutation [117]. This could be explained, at least in part, by the type of alterations in the SERPING1 gene and mutations in other genes encoding proteins that are part of the complement, fibrinolysis, coagulation, and kinin systems [99]. The involvement of epigenetic changes [118], viruses, and colonizing microorganisms [119], as well as environmental factors in the pathogenesis of HAE, has also been postulated [61]. As genotype-phenotype correlations in HAE have been reviewed in detail by Loli-Ausejo et al. in the current issue, we only provide a few representative examples of studies with important implications for the discovery of prognostic biomarkers. Several studies investigated correlations between the type of mutations in the SERPINGl gene (including nonsense, frameshift, large deletions or insertions, splicing defect, and missense mutations) and the clinical course of C1-INH-HAE. Only patients carrying missense mutations leading to the change of a single amino acid exhibited a less severe clinical phenotype [120-122]. However, the results concerning the effect of these mutations on the onset of HAE were conflicting. Bors et al. [120] and Speletas et al. [121] found that the first symptoms of C1-INH-HAE appeared at an older age in patients harboring missense mutations. Several other studies did not find a correlation between different types of mutations and clinical phenotype, but the size of the patient population was relatively small [34, 123, 124].

Disease-modifying factors, such as $F 12$ or KLKB1 gene polymorphisms, have been studied in the context of the clinical variability of C1-INH-HAE or nC1-INH-HAE. The c. $-4 \mathrm{C} / \mathrm{T}$ polymorphism (rs1801020) in the 5-UTR region of the $F 12$ gene was associated with a significantly delayed disease onset [120,125,126], regardless of the type of SERPING1 mutations [125]. Moreover, the T allele was more common in asymptomatic patients [127]. An association between the c.428G/A (rs3733402) polymorphism in the $K L K B 1$ gene, encoding $\mathrm{PKa}$, and clinical variability of $\mathrm{C} 1-$ INH-HAE was also investigated. Gianni et al. [128] showed that patients carrying $\mathrm{G}$ allele exhibited a delayed onset of HAE. The disease onset was further delayed in individuals harboring both c. $-4 \mathrm{C} / \mathrm{T}$ and c.428G/A polymorphisms. No associations were found between the type of SERPING1 mutations and age at disease onset. More recently, the F12 c.-4C/T polymorphism has been linked with FXII-HAE (p.Thr309Lys variant). Patient carrying the c.-4CC genotype 
showed higher PKa-like activity and exhibited more severe and frequent manifestations of the disease [129].

\section{Future Research Directions}

Future applications and research directions in the field are summarized in Fig. 1. A combination of several different biomarkers can also be used. Such an approach may increase overall performance, especially when investigated biomarkers reflect different underlying pathways of HAE. Pharmacogenomic predictive biomarkers might identify individuals who are or are not likely to respond to treatment and thus guide the use of a targeted therapy. Therefore, a discovery of such potential biomarkers would have important implications for therapeutic recommendations reflecting the precision medicine paradigm ("the right drug to the right patient") [12]. Moreover, biomarkers may serve as validated surrogate endpoints in clinical trials if there is a strong mechanistic rationale and clinical evidence for a possible correlation between a change in biomarker levels and a specific clinical endpoint [11]. Furthermore, validated biomarkers could provide consistent and objective outcome measure and thus facilitate a comparison of different intervention strategies and clinical trial results.

\section{Conclusions}

In conclusion, validated biomarkers could enable a precise diagnosis and personalized management as well as facilitate clinical trials in HAE. New technologies may help establish such biomarkers together with laboratory tests for their detection and measurement, but there is an urgent need to conduct more well-designed studies or complete the ongoing ones in order to obtain solid evidence for their usefulness.

Author Contribution Concept and design: AR, GP. Acquisition of data: AR, GP, MK. Drafting of the manuscript: GP, MK.

Open Access This article is licensed under a Creative Commons Attribution 4.0 International License, which permits use, sharing, adaptation, distribution and reproduction in any medium or format, as long as you give appropriate credit to the original author(s) and the source, provide a link to the Creative Commons licence, and indicate if changes were made. The images or other third party material in this article are included in the article's Creative Commons licence, unless indicated otherwise in a credit line to the material. If material is not included in the article's Creative Commons licence and your intended use is not permitted by statutory regulation or exceeds the permitted use, you will need to obtain permission directly from the copyright holder. To view a copy of this licence, visit http://creativecommons.org/licenses/by/4.0/.

\section{References}

1. ClinicalTrials.gov. https://clinicaltrials.gov/. Accessed Nov 15, 2020

2. PubMed. https://pubmed.ncbi.nlm.nih.gov/. Accessed Nov 15, 2020

3. Bakker DS, Ariens LFM, Giovannone B et al (2020) EASI p-EASI: predicting disease severity in atopic dermatitis patients treated with dupilumab using a combination of serum biomarkers. Allergy 75(12):3287-3289

4. Barker-Tejeda TC, Bazire R, Obeso D et al (2020) Exploring novel systemic biomarker approaches in grass-pollen sublingual immunotherapy using omics. Allergy in press

5. Buhl R, Korn S, Menzies-Gow A et al (2020) Prospective, single-arm, longitudinal study of biomarkers in real-world patients with severe asthma. J Allergy Clin Immunol Pract 8(8):2630-2639.e6

6. Nieto-Fontarigo JJ, González-Barcala FJ, Andrade-Bulos LJ et al (2020) iTRAQ-based proteomic analysis reveals potential serum biomarkers of allergic and nonallergic asthma. Allergy in press

7. Santos AF, du Toit G, O'Rourke C et al (2020) Biomarkers of severity and threshold of allergic reactions during oral peanut challenges. J Allergy Clin Immunol 146(2):344-355

8. Khan DA, Phillips EJ (2020) Pharmacogenomic biomarkers in allergy and immunology practice. J Allergy Clin Immunol 146(3):509-512

9. Busse PJ, Christiansen SC (2020) Hereditary angioedema. N Engl J Med 382(12):1136-1148

10. Busse PJ, Christiansen SC, Riedl MA et al (2020) US HAEA Medical Advisory Board 2020 guidelines for the management of hereditary angioedema. J Allergy Clin Immunol Pract S2213-2198(20):30878-30883

11. FDA-NIH Biomarker Working Group (2017) BEST ( Biomarkers, EndpointS, and other Tools ). https://www.ncbi.nlm.nih.gov/ books/NBK326791/

12. Ferlini A, Scotton C, Novelli G (2013) Biomarkers in rare diseases. Public Health Genomics 16(6):313-321

13. Kaplan AP, Maas C (2017) The search for biomarkers in hereditary angioedema. Front Med (Lausanne) 4:206

14. Germenis AE, Cicardi M (2019) Driving towards precision medicine for angioedema without wheals. J Autoimmun 104:102312

15. Deroux A, Vilgrain I, Dumestre-Pérard C et al (2015) Towards a specific marker for acute bradykinin-mediated angioedema attacks: a literature review. Eur J Dermatol 25(4):290-295

16. Farkas H, Veszeli N, Kajdácsi E et al (2016) "Nuts and Bolts" of laboratory evaluation of angioedema. Clin Rev Allergy Immunol 51(2):140-151

17. Christiansen SC, Zuraw BL (2017) Laboratory approaches for assessing contact system activation. Immunol Allergy Clin North Am 37(3):527-539

18. Wagenaar-Bos IGA, Drouet C, Aygören-Pursun E et al (2008) Functional C1-Inhibitor diagnostics in hereditary angioedema: assay evaluation and recommendations. J Immunol Methods 338(1-2):14-20

19. Li HH, Busse P, Lumry WR et al (2015) Comparison of chromogenic and ELISA functional $\mathrm{C} 1$ inhibitor tests in diagnosing hereditary angioedema. J Allergy Clin Immunol Pract 3(2):200-205

20. Gompels MM, Lock RJ, Morgan JE et al (2002) A multicentre evaluation of the diagnostic efficiency of serological investigations for $\mathrm{C} 1$ inhibitor deficiency. J Clin Pathol 55(2): 145-147

21. Zeerleder S (2011) C1-inhibitor: more than a serine protease inhibitor. Semin Thromb Hemost 37(4):362-374 
22. Charest-Morin X, Betschel S, Borici-Mazi R et al (2018) The diagnosis of hereditary angioedema with $\mathrm{C} 1$ inhibitor deficiency: a survey of Canadian physicians and laboratories. Allergy Asthma Clin Immunol 14:83

23. Veronez CL, Grumach AS (2020) Angioedema without urticaria: novel findings which must be measured in clinical setting. Curr Opin Allergy Clin Immunol 20(3):253-260

24. Tarzi MD, Hickey A, Förster T et al (2007) An evaluation of tests used for the diagnosis and monitoring of $\mathrm{C} 1$ inhibitor deficiency: normal serum $\mathrm{C} 4$ does not exclude hereditary angio-oedema. Clin Exp Immunol 149(3):513-516

25. Agostini A (1989) Inherited C1 inhibitor deficiency. Complement Inflamm 6(2):112-118

26. Ziccardi RJ (1982) A new role for C-1-inhibitor in homeostasis: control of activation of the first component of human complement. J Immunol 128(6):2505-2508

27. Joseph K, Bains S, Tholanikunnel BG et al (2015) A novel assay to diagnose hereditary angioedema utilizing inhibition of bradykinin-forming enzymes. Allergy 70(1):115-119

28. Lai Y, Zhang G, Zhou Z et al (2020) A novel functional C1 inhibitor activity assay in dried blood spot for diagnosis of hereditary angioedema. Clin Chim Acta 504:155-162

29. Suffritti C, Zanichelli A, Maggioni L et al (2014) Highmolecular-weight kininogen cleavage correlates with disease states in the bradykinin-mediated angioedema due to hereditary C1-inhibitor deficiency. Clin Exp Allergy 44(12):1503-1514

30. Kajdácsi E, Jani PK, Csuka D et al (2014) Endothelial cell activation during edematous attacks of hereditary angioedema types I and II. J Allergy Clin Immunol 133(6):1686-1691

31. Cugno M, Zanichelli A, Bellatorre AG et al (2009) Plasma biomarkers of acute attacks in patients with angioedema due to C1-inhibitor deficiency. Allergy 64(2):254-257

32. Kelemen Z, Moldovan D, Mihály E et al (2010) Baseline level of functional C1-inhibitor correlates with disease severity scores in hereditary angioedema. Clin Immunol 134(3):354-358

33. Csuka D, Füst G, Farkas H, Varga L (2011) Parameters of the classical complement pathway predict disease severity in hereditary angioedema. Clin Immunol 139(1):85-93

34. Bafunno V, Bova M, Loffredo S et al (2014) Mutational spectrum of the $\mathrm{C} 1$ inhibitor gene in a cohort of Italian patients with hereditary angioedema: description of nine novel mutations. Ann Hum Genet 78(2):73-82

35. Longhurst H, Cicardi M, Craig T et al (2017) Prevention of hereditary angioedema attacks with a subcutaneous $\mathrm{C} 1$ inhibitor. N Engl J Med 376(12):1131-1140

36. Hack CE, Relan A, van Amersfoort ES, Cicardi M (2012) Target levels of functional C1-inhibitor in hereditary angioedema. Allergy 67(1):123-130

37. Kaplan AP, Pawaskar D, Chiao J (2020) C1 inhibitor activity and angioedema attacks in patients with hereditary angioedema. J Allergy Clin Immunol Pract 8(3):892-900

38. Betschel S, Badiou J, Binkley K et al (2019) The International/ Canadian Hereditary Angioedema Guideline. Allergy Asthma Clin Immunol 15:72

39. Spath PJ, Wuthrich B, Butler R (1984) Quantification of $\mathrm{C} 1$-inhibitor functional activities by immunodiffusion assay in plasma of patients with hereditary angioedema - evidence of a functionally critical level of $\mathrm{C} 1$ - inhibitor concentration. Complement 1(3):147-159

40. Cugno M, Nuijens J, Hack E et al (1990) Plasma levels of $\mathrm{C} 1$ inhibitor complexes and cleaved $\mathrm{Ct}$ inhibitor in patients with hereditary angioneurotic edema. J Clin Invest 85(4):1215-1220

41. Cugno M, Hack CE, de Boer JP et al (1993) Generation of plasmin during acute attacks of hereditary angioedema. J Lab Clin Med 121(1):38-43
42. Cicardi M, Bergamaschini L, Cugno M et al (1991) Long-term treatment of hereditary angioedema with attenuated androgens: a survey of a 13-year experience. J Allergy Clin Immunol 87(4):768-773

43. Spaeth P, Wüthrich B (1998) Inherited and acquired deficiencies of $\mathrm{C} 1$ esterase inhibitor in humans. In: Rother K, Till GO, Hausch GM (eds) The Complement System. Springer-Verlag, Berlin Heidelberg, pp 353-410

44. Varga L, Széplaki G, Visy B et al (2007) C1-inhibitor (C1-INH) autoantibodies in hereditary angioedema. Strong correlation with the severity of disease in C1-INH concentrate naïve patients. Mol Immunol 44(6): 1454-60

45. Hansen CB, Csuka D, Munthe-Fog L et al (2015) The levels of the lectin pathway serine protease MASP-1 and its complex formation with $\mathrm{C} 1$ inhibitor are linked to the severity of hereditary angioedema. J Immunol 195(8):3596-3604

46. Csuka D, Munthe-Fog L, Hein E et al (2014) Activation of the ficolin-lectin pathway during attacks of hereditary angioedema. J Allergy Clin Immunol 134(6):1388-1393.e1

47. Cicardi M, Zuraw BL (2018) Angioedema due to bradykinin dysregulation. J Allergy Clin Immunol Pract 6(4):1132-1141

48. Nussberger J, Cugno M, Amstutz C et al (1998) Plasma bradykinin in angio-oedema. Lancet 351(9117):1693-1697

49. Nussberger J, Cugno M, Cicardi M, Agostoni A (1999) Local bradykinin generation in hereditary angioedema. J Allergy Clin Immunol 104(6):1321-1322

50. Seip KF, Bjerknes KC, Johansen HT et al (2014) Bradykinin analysis revived - a validated method for determination of its stable metabolite in whole blood by LC-MS/MS. J Chromatogr B Analyt Technol Biomed Life Sci 947-948:139-144

51. Pietzner M, Kaul A, Henning AK et al (2017) Comprehensive metabolic profiling of chronic low-grade inflammation among generally healthy individuals. BMC Med 15(1):210

52. Hofman ZLM, de Maat S, van Doorn C et al (2017) Cleaved kininogen as a biomarker for bradykinin release in hereditary angioedema. J Allergy Clin Immunol 140(6):1700-1703.e8

53. Zhang G, Sexton DJ, Faucette RR et al (2017) 2D-LC-MS/MS to measure cleaved high-molecular-weight kininogen in human plasma as a biomarker for C1-INH-HAE. Bioanalysis 9(19):1477-1491

54. Cugno M, Cicardi M, Coppola R, Agostoni A (1996) Activation of the factor XII and cleavage of high molecular weight kininogen during acute attacks in hereditary and acquired $\mathrm{C} 1$-inhibitor deficiencies. Immunopharmacology 33(1-3):361-364

55. Cugno M, Cicardi M, Bottasso B et al (1997) Activation of the coagulation cascade in $\mathrm{C} 1$-inhibitor deficiencies. Blood 89(9):3213-3218

56. Banerji A, Busse P, Shennak M et al (2017) Inhibiting plasma kallikrein for hereditary angioedema prophylaxis. N Engl J Med 376(8):717-728

57. Bova M, Suffritti C, Bafunno V et al (2020) Impaired control of the contact system in hereditary angioedema with normal C1-inhibitor. Allergy 75(6):1394-1403

58. Joseph K, Tuscano TB, Kaplan AP (2008) Studies of the mechanisms of bradykinin generation in hereditary angioedema plasma. Ann Allergy Asthma Immunol 101(3):279-286

59. Lara-Marquez ML, Christiansen SC, Riedl MA et al (2018) Threshold-stimulated kallikrein activity distinguishes bradykinin- from histamine-mediated angioedema. Clin Exp Allergy 48(11):1429-1438

60. Csuka D, Veszeli N, Imreh É et al (2015) Comprehensive study into the activation of the plasma enzyme systems during attacks of hereditary angioedema due to C1-inhibitor deficiency. Orphanet J Rare Dis 10:132

61. Cichon S, Martin L, Hennies HC et al (2006) Increased activity of coagulation factor XII (Hageman Factor) causes hereditary angioedema type III. Am J Hum Genet 79(6):1098-1104 
62. Bork K, Kleist R, Hardt J, Witzke G (2009) Kallikrein-kinin system and fibrinolysis in hereditary angioedema due to factor XII gene mutation Thr309Lys. Blood Coagul Fibrinolysis 20(5):325-332

63. Konings J, Cugno M, Suffritti C et al (2013) Ongoing contact activation in patients with hereditary angioedema. PLoS One 8(8):e74043

64. Dessart P, Defendi F, Humeau H et al (2015) Distinct conditions support a novel classification for bradykininmediated angio-oedema. Dermatology 230(4):324-331

65. Drouet C, Désormeaux A, Robillard J et al (2008) Metallopeptidase activities in hereditary angioedema: effect of androgen prophylaxis on plasma aminopeptidase P. J Allergy Clin Immunol 121(2):429-433

66. Charignon D, Ghannam A, Defendi F et al (2014) Hereditary angioedema with F12 mutation: factors modifying the clinical phenotype. Allergy 69(12):1659-1665

67. Defendi F, Charignon D, Ghannam A et al (2013) Enzymatic assays for the diagnosis of bradykinin-dependent angioedema. PLoS One 8(8):e70140

68. van Geffen M, Cugno M, Lap P et al (2012) Alterations of coagulation and fibrinolysis in patients with angioedema due to C1-inhibitor deficiency. Clin Exp Immunol 167(3):472-478

69. Waage Nielsen E, Thidemann Johansen H, Høgåsen K et al (1996) Activation of the complement, coagulation, fibrinolytic and kallikrein-kinin systems during attacks of hereditary angioedema. Scand J Immunol 44(2):185-192

70. Joseph K, Tholanikunnel BG, Wolf B et al (2016) Deficiency of plasminogen activator inhibitor 2 in plasma of patients with hereditary angioedema with normal $\mathrm{C} 1$ inhibitor levels. J Allergy Clin Immunol 137(6):1822-1829.e1

71. Marlu R, Deroux A, Du-Thanh A et al (2017) Normal PAI-2 level in French FXII-HAE patients. J Allergy Clin Immunol 139(5):1719-1720

72. Deroux A, Dumestre-Perard C, Khalil-Mgharbel A et al (2016) BIOBRAD Study: the search for biomarkers of bradykininmediated angio-oedema attacks. Int Arch Allergy Immunol 170(2):108-114

73. Reshef A, Zanichelli A, Longhurst $\mathrm{H}$ et al (2015) Elevated D-dimers in attacks of hereditary angioedema are not associated with increased thrombotic risk. Allergy 70(5):506-513

74. Reshef A, Levy D, Longhurst $\mathrm{H}$ et al (2020) Effects of continuous plasma-derived subcutaneous $\mathrm{C} 1$-esterase inhibitor on coagulation and fibrinolytic parameters. Thromb Haemost in press

75. Bounds EJ, Kok SJ (2020) D Dimer. In: StatPearls [Internet], StatPearls Publishing LLC

76. Bouillet L, Mannic T, Arboleas M et al (2011) Hereditary angioedema: key role for kallikrein and bradykinin in vascular endothelial-cadherin cleavage and edema formation. J Allergy Clin Immunol 128(1):232-234

77. Czícz J, Schaffer G, Csuka D et al (2012) Endothelial cell function in patients with hereditary angioedema: elevated soluble E-selectin level during inter-attack periods. J Clin Immunol 32(1):61-69

78. Kajdácsi E, Jani PK, Csuka D et al (2016) Novel vasoregulatory aspects of hereditary angioedema: the role of arginine vasopressin, adrenomedullin and endothelin-1. J Clin Immunol 36(2):160-170

79. Kajdácsi E, Varga L, Prohászka Z et al (2016) Atrial natriuretic peptide as a novel biomarker of hereditary angioedema. Clin Immunol 165:45-46

80. Demirturk M, Akpinar TS, Kose M et al (2017) Endocan: a novel marker of endothelial dysfunction in C1-inhibitordeficient hereditary angioedema. Int Arch Allergy Immunol 174(2):104-107
81. Loffredo S, Bova M, Suffritti C et al (2016) Elevated plasma levels of vascular permeability factors in $\mathrm{C} 1$ inhibitor-deficient hereditary angioedema. Allergy 71(7):989-996

82. Ferrara AL, Bova M, Petraroli A et al (2020) Hereditary angioedema attack: what happens to vasoactive mediators? Int Immunopharmacol 78:106079

83. Loffredo S, Ferrara AL, Bova M et al (2018) Secreted phospholipases A2 in hereditary angioedema with C1-inhibitor deficiency. Front Immunol 9:1721

84. de Nucci G, Gryglewski RJ, Warner TD, Vane JR (1988) Receptor-mediated release of endothelium-derived relaxing factor and prostacyclin from bovine aortic endothelial cells is coupled. Proc Natl Acad Sci U S A 85(7):2334-2338

85. Zuraw BL, Christiansen SC (2016) HAE pathophysiology and underlying mechanisms. Clin Rev Allergy Immunol 51(2):216-229

86. Bas M, Storck K, Strassen U (2017) Potential biomarkers for the diagnosis of angiotensin-converting enzyme inhibitor-induced angioedema. ORL J Otorhinolaryngol Relat Spec 79(1-2):85-92

87. Demirtürk M, Gelincik A, Çinar S et al (2014) Increased eNOS levels in hereditary angioedema. Int Immunopharmacol 20(1):264-268

88. Firinu D, Bassareo PP, Zedda AM et al (2018) Impaired endothelial function in hereditary angioedema during the symptom-free period. Front Physiol 9:523

89. del Giacco SR, Firinu D, Minciullo PL et al (2019) Oxidative stress markers in patients with hereditary angioedema. Arch Med Sci 15(1):92-98

90. Obtułowicz K, Góralska J, Bogdali A et al (2020) Bradykinin and oxidative stress in patients with hereditary angioedema due to $\mathrm{C} 1$ inhibitor deficiency. Pol Arch Intern Med 130(2):79-88

91. Hofman ZLM, Relan A, Hack CE (2014) C-reactive protein levels in hereditary angioedema. Clin Exp Immunol 177(1):280-286

92. Veszeli N, Csuka D, Zotter Z et al (2015) Neutrophil activation during attacks in patients with hereditary angioedema due to C1-inhibitor deficiency. Orphanet J Rare Dis 10:156

93. Márkus B, Veszeli N, Temesszentandrási G et al (2019) Serum fetuin-A, tumor necrosis factor alpha and C-reactive protein concentrations in patients with hereditary angioedema with C1-inhibitor deficiency. Orphanet J Rare Dis 14(1):67

94. Arcoleo F, Salemi M, la Porta A et al (2014) Upregulation of cytokines and IL-17 in patients with hereditary angioedema. Clin Chem Lab Med 52(5):e91-e93

95. Salemi M, Mandalà V, Muggeo V et al (2016) Growth factors and IL-17 in hereditary angioedema. Clin Exp Med 16(2):213-218

96. Visy B, Fust G, Varga L et al (2004) Sex hormones in hereditary angioneurotic oedema. Clin Endocrinol (Oxf) 60(4):508-515

97. Larrauri B, Hester CG, Jiang $\mathrm{H}$ et al (2020) sgp120 and the contact system in hereditary angioedema: a diagnostic tool in HAE with normal C1 inhibitor. Mol Immunol 119:27-34

98. Food and Drug Administration, HHS. International Conference on Harmonisation; Guidance on E15 Pharmacogenomics Definitions and Sample Coding; Availability. Notice. Fed Regist 73(68):19074-6

99. Germenis AE, Speletas M (2016) Genetics of hereditary angioedema revisited. Clin Rev Allergy Immunol 51(2):170-182

100. Germenis AE, Margaglione M, Pesquero JB et al (2020) International consensus on the use of genetics in the management of hereditary angioedema. J Allergy Clin Immunol Pract 8(3):901-911

101. Nielsen EW, Johansen HT, Holt J, Mollnes TE (1994) C1 inhibitor and diagnosis of hereditary angioedema in newborns. Pediatr Res 35(2):184-187

102. Maurer M, Magerl M, Ansotegui I et al (2018) The international $\mathrm{WAO} / \mathrm{EAACI}$ guideline for the management of hereditary angioedema - the 2017 revision and update. Allergy 73(8):1575-1596

103. Dewald G, Bork K (2006) Missense mutations in the coagulation factor XII (Hageman factor) gene in hereditary angioedema 
with normal $\mathrm{C} 1$ inhibitor. Biochem Biophys Res Commun 343(4):1286-1289

104. Bork K, Wulff K, Steinmüller-Magin L et al (2018) Hereditary angioedema with a mutation in the plasminogen gene. Allergy 73(2):442-450

105. Bafunno V, Firinu D, D'Apolito M et al (2018) Mutation of the angiopoietin-1 gene (ANGPT1) associates with a new type of hereditary angioedema. J Allergy Clin Immunol 141(3):1009-1017

106. Zuraw BL (2018) Hereditary angioedema with normal C1 inhibitor: four types and counting. J Allergy Clin Immunol 141(3):884-885

107. SERPING1 serpin family $G$ member 1 [Homo sapiens (human)] - Gene - NCBI. https://www.ncbi.nlm.nih.gov/ gene $? \mathrm{Db}=$ gene $\& \mathrm{Cmd}=$ DetailsSearch $\&$ Term $=710$. Accessed 7 Nov 2020

108. Bork K, Wulff K, Meinke P et al (2011) A novel mutation in the coagulation factor 12 gene in subjects with hereditary angioedema and normal C1-inhibitor. Clin Immunol 141(1):31-35

109. Kiss N, Barabás E, Várnai K et al (2013) Novel duplication in the F12 gene in a patient with recurrent angioedema. Clin Immunol 149(1):142-145

110. d'Apolito M, Santacroce R, Colia AL et al (2019) Angiopoietin-1 haploinsufficiency affects the endothelial barrier and causes hereditary angioedema. Clin Exp Allergy 49(5):626-635

111. Dewald G (2018) A missense mutation in the plasminogen gene, within the plasminogen kringle 3 domain, in hereditary angioedema with normal $\mathrm{C} 1$ inhibitor. Biochem Biophys Res Commun 498(1):193-198

112. Bork K, Wulff K, Rossmann H et al (2019) Hereditary angioedema cosegregating with a novel kininogen 1 gene mutation changing the $\mathrm{N}$-terminal cleavage site of bradykinin. Allergy 74(12):2479-2481

113. Ariano A, D’Apolito M, Bova M et al (2020) A myoferlin gainof-function variant associates with a new type of hereditary angioedema. Allergy 75(11):2989-2992

114. Cagini N, Veronez CL, Azevedo BF et al (2018) In silico analysis of alterations in $A N G P T 1$ gene supports a new pathway responsible to mediate hereditary angioedema in Brazilian patients with no mutations in SERPING1 and F12 genes. J Allergy Clin Immunol 141:AB46

115. Maas C (2019) Plasminflammation - an emerging pathway to bradykinin production. Front Immunol 10:2046

116. Bork K, Zibat A, Ferrari DM et al (2020) Hereditary angioedema in a single family with specific mutations in both plasminogen and SERPING1 genes. J Dtsch Dermatol Ges 18(3):215-223

117. Wu MA, Perego F, Zanichelli A, Cicardi M (2016) Angioedema phenotypes: disease expression and classification. Clin Rev Allergy Immunol 51(2):162-169
118. Khan S, Longhurst H (2020) Epigenetic alterations on C1-inhibitor expression may influence hereditary angioedema attack frequency and C4 levels. Clin Exp Immunol 202(2):144-145

119. Kaplan AP, Greaves MW (2005) Angioedema. J Am Acad Dermatol 53(3):373-388

120. Bors A, Csuka D, Varga L et al (2013) Less severe clinical manifestations in patients with hereditary angioedema with missense C1INH gene mutations. J Allergy Clin Immunol 131(6):1708-1711

121. Speletas M, Szilagyi A, Psarros F et al (2015) Hereditary angioedema: molecular and clinical differences among European populations. J Allergy Clin Immunol 135(2):570-573

122. Andrejević S, Korošec P, Šilar M et al (2015) Hereditary angioedema due to $\mathrm{C} 1$ inhibitor deficiency in Serbia: two novel mutations and evidence of genotype-phenotype association. PLoS One 10(11):e0142174

123. Xu YY, Zhi YX, Yin J et al (2012) Mutational spectrum and geno-phenotype correlation in Chinese families with hereditary angioedema. Allergy 67(11):1430-1436

124. Maia LSM, Moreno AS, Ferriani MPL et al (2019) Genotypephenotype correlations in Brazilian patients with hereditary angioedema due to $\mathrm{C} 1$ inhibitor deficiency. Allergy 74(5):1013-1016

125. Speletas M, Szilágyi CD et al (2015) F12-46C/T polymorphism as modifier of the clinical phenotype of hereditary angioedema. Allergy 70(12):1661-1664

126. Grivčeva-Panovska V, Košnik M, Korošec P et al (2018) Hereditary angioedema due to $\mathrm{C} 1$-inhibitor deficiency in Macedonia: clinical characteristics, novel SERPING1 mutations and genetic factors modifying the clinical phenotype. Ann Med 50(3):269-276

127. Rijavec M, Košnik M, Andrejević $S$ et al (2019) The functional promoter F12-46C/T variant predicts the asymptomatic phenotype of C1-INH-HAE. Clin Exp Allergy 49(11):1520-1522

128. Gianni P, Loules G, Zamanakou M et al (2017) Genetic determinants of $\mathrm{C} 1$ inhibitor deficiency angioedema age of onset. Int Arch Allergy Immunol 174(3-4):200-204

129. Corvillo F, Morena-Barrio ME de la, Marcos-Bravo C et al (2020) The FXII c.-4T $>$ C polymorphism as a disease modifier in patients with hereditary angioedema due to the FXII p.Thr328Lys variant. Front Genet 11:1033

Publisher's Note Springer Nature remains neutral with regard to jurisdictional claims in published maps and institutional affiliations. 